\title{
An unusual focal atrial tachycardia that simulates a common node reentrant tachycardia: A case report
}

\author{
Carlo Domenico Maida, Anna Cirrincione, Mario Daidone, Valerio Vassallo, Alessandro Del Cuore, Antonino \\ Tuttolomondo, Antonio Pinto \\ Dipartimento Biomedico di Medicina Interna e Specialistica, U.O.C di Medicina Interna e Cardioangiologia, Università degli \\ Studi di Palermo, Palermo, Italy
}

Received: August 5, 2017

Accepted: October 15, 2017

Online Published: October 20, 2017

DOI: $10.5430 /$ crim.v4n4p33

URL: https://doi.org/10.5430/crim.v4n4p33

\begin{abstract}
Focal atrial tachycardia is a relatively uncommon paroxysmal supraventricular tachycardia. Although atrioventricular conduction is regularly 1:1 and the PR interval is often in the normal range, occasionally a 1:1 atrial tachycardia may have a short RP interval, especially when the heart rate is particularly high or the atrioventricular conduction is markedly increased. In these cases, it is necessary to differentiate focal atrial tachycardia from common atrioventricular nodal reentrant tachycardia, which is the most frequent form of paroxysmal supraventricular tachycardia. We describe a case of unusual focal atrial tachycardia with a short RP interval $(<90 \mathrm{~ms})$ in a patient with a marked AV first-degree block which simulates a typical atrioventricular nodal reentrant tachycardia.
\end{abstract}

Key Words: Focal atrial tachycardia, Atrioventricular nodal reentrant tachycardia, Paroxysmal supraventricular tachycardia, Holter monitoring

\section{INTRODUCTION}

Atrial tachycardia (AT) is quite rare, representing 5\%-15\% of all supraventricular tachycardias. It is common in elderly patients and in particular in patients with multiple comorbidities, which represent the typical patients of internal medicine wards. Differential diagnosis should be made with the other types of supraventricular tachycardias (SVTs), including sinus tachycardia, atrial flutter and atrioventricular (AV) junction-dependent reentrant tachycardias (AV nodal reentrant tachycardia and $\mathrm{AV}$ reentrant tachycardia using an accessory pathway). The evaluation of the association between P waves and QRS complex may be useful for the diagnosis. Usually the AV conduction is $1: 1$ and the PR interval is in the normal range but sometimes a 1:1 atrial tachycardia can manifest itself with a short RP interval (<
$90 \mathrm{~ms}$ ), in particular when a long first-degree AV block is present at the same time. In these cases, it is essential to discriminate focal AT from common atrioventricular nodal reentrant tachycardia (AVNRT), which is the most frequent type of paroxysmal SVTs. Differentiating among these diagnoses requires electrocardiographic analysis and additional examination, such as vagal stimulation (e.g., carotid sinus massage and Valsalva maneuver), or adenosine infusion. Additional details to distinguish AT from other SVTs can be provided by Holter ECG analysis, although it is often overlooked.

\section{Case presentation}

A 90-year-old female patient, affected by hypertension, chronic cerebrovascular disease and diastolic heart failure,

\footnotetext{
* Correspondence: Carlo Domenico Maida; Email: carlodomenico.maida@ hotmail.com; Address: Dipartimento Biomedico di Medicina Interna e Specialistica, Università degli Studi di Palermo, P.zza delle Cliniche n.2, 90127 Palermo, Italy.
}

Published by Sciedu Press 
was admitted to our unit for the diagnostic evaluation of symptomatology characterized by palpitations and shortness of breath, which occurred several times per year, lasting 5-10 minutes. Her admission 12 lead electrocardiogram (ECG) showed a regular sinus rhythm, ventricular bigeminy and a marked first degree AV block (see Figure 1). Echocardiography showed mild wall thickening and mild diastolic dysfunction. At first, an ischemic heart disease was ruled out. Few days later a Holter ECG monitoring was performed. This examination showed a regular supraventricular tachycardia (QRS < $120 \mathrm{~ms}$ ) of abrupt onset at a ventricular rate of about 150 beats per minute triggered by a single atrial premature beat with a prolongation of PR interval (see Figure 2). Looking at the electrocardiographic trace, $P$ waves were inscribed at the end of the QRS complex, seen best in the inferior leads and as a slightly positive $\mathrm{R}$ (pseudo $\mathrm{r}$ prime) in lead V1. RP interval was shorter than PR interval and it was lower than $90 \mathrm{~ms}$. The diagnosis seemed to be typical AVNRT. Looking immediately after in the ECG trace however, PR and RP intervals became slightly irregular and PR interval progressively increased until a $P$ wave was blocked and remained concealed by the QRS complex configuring the Wenckebach phenomenon (see Figure 3). Subsequently afterwards, maybe due to the increased refractoriness of the AV node, the AV conduction became 2:1 (see Figure 4) and finally tachycardia ended abruptly. The Wenckebach conduction during a SVT can not be found during typical AVNRT and allowed the diagnosis of AT. Patient was discharged with medical therapy (oral diltiazem) with an improvement of the symptomatology to follow up control.

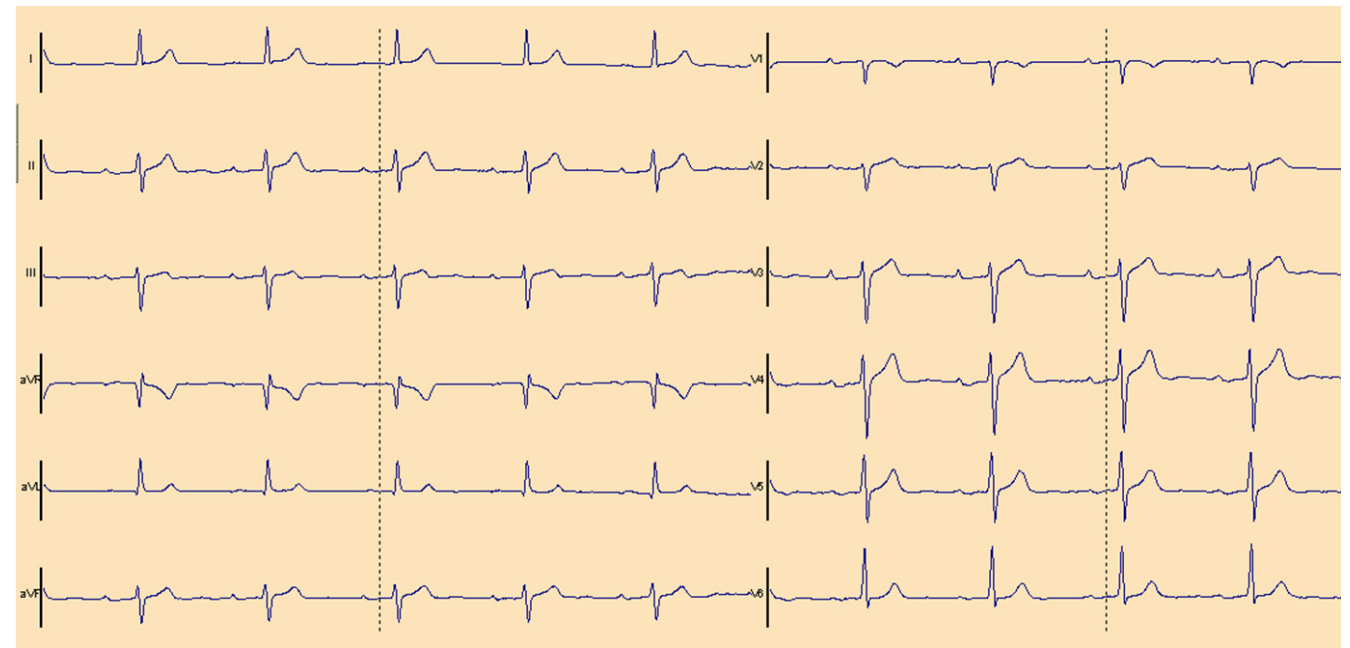

Figure 1. Twelve lead electrocardiogram trace performed at the admission of the patient

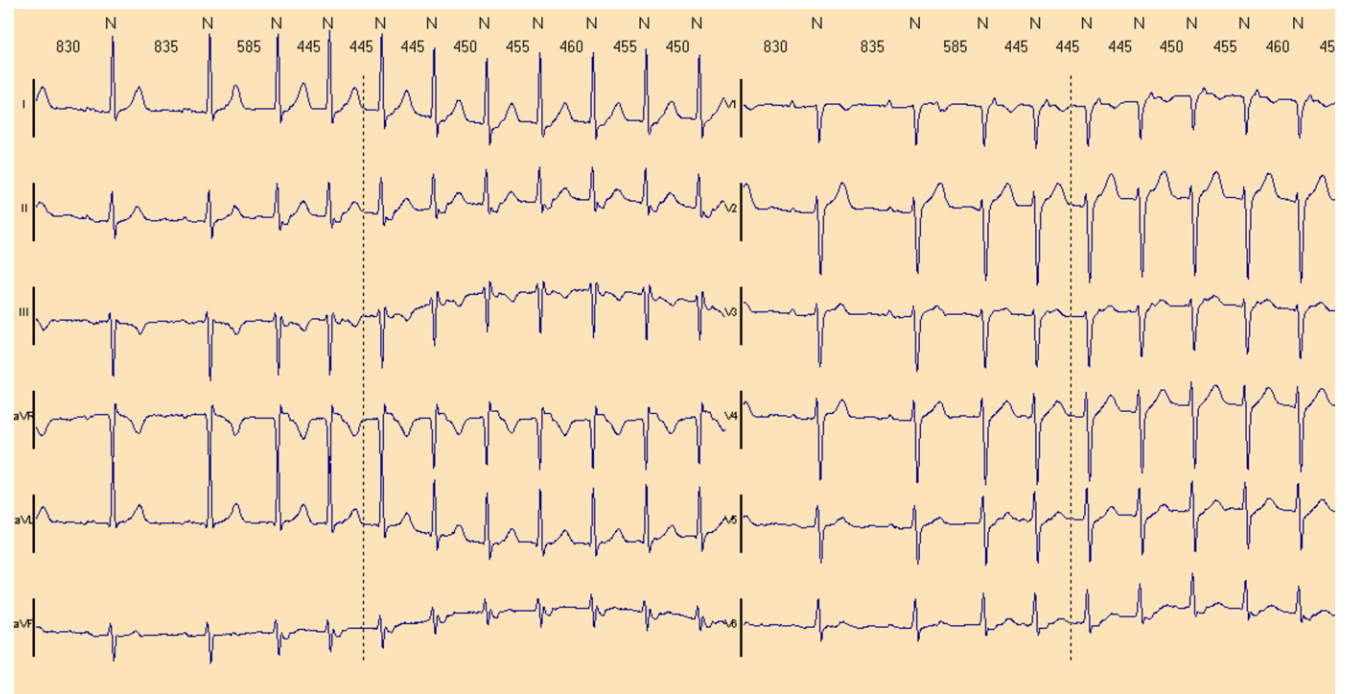

Figure 2. Regular supraventricular tachycardia (QRS $<120 \mathrm{~ms}$ ) of abrupt onset triggered by a single atrial premature beat with a prolongation of PR interval. Should be noted that the wave P generating the supraventricular tachycardia appears to be of altered morphology as it is embedded in wave $\mathrm{T}$ of the previous complex. 


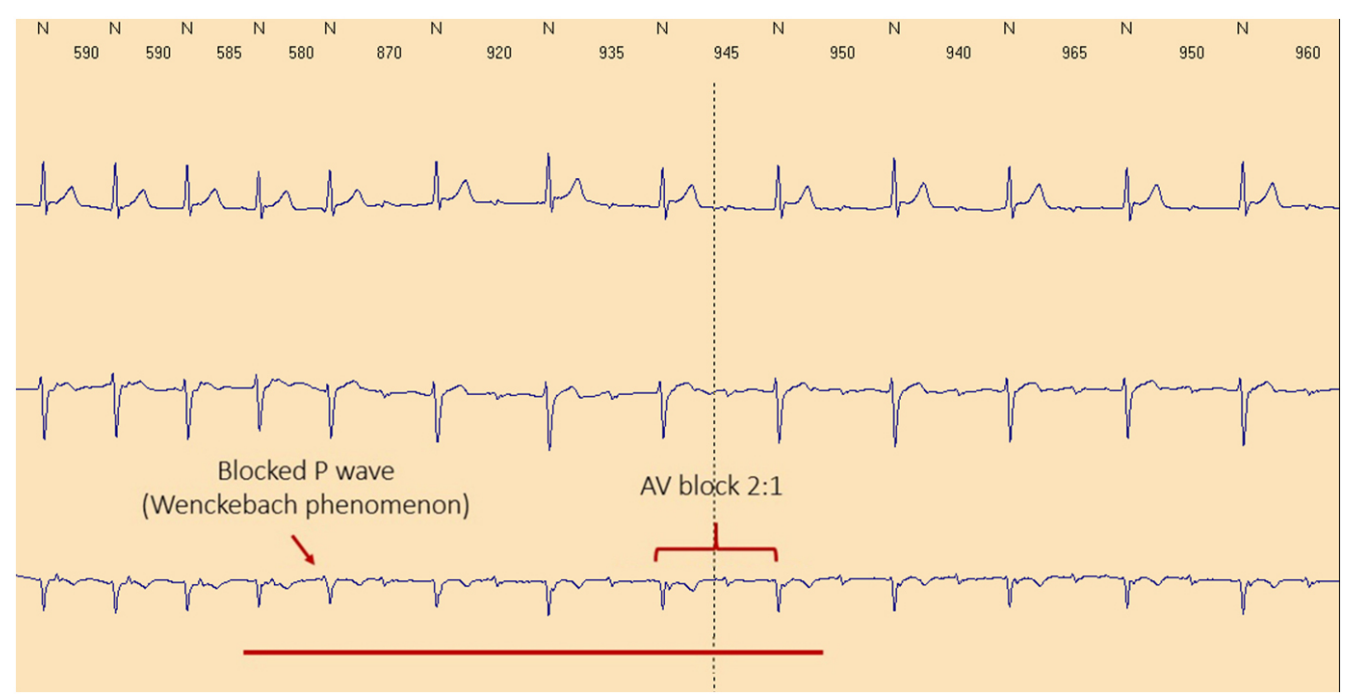

Figure 3. Wenckebach phenomenon at Holter monitoring allows the diagnosis of atrial tachycardia

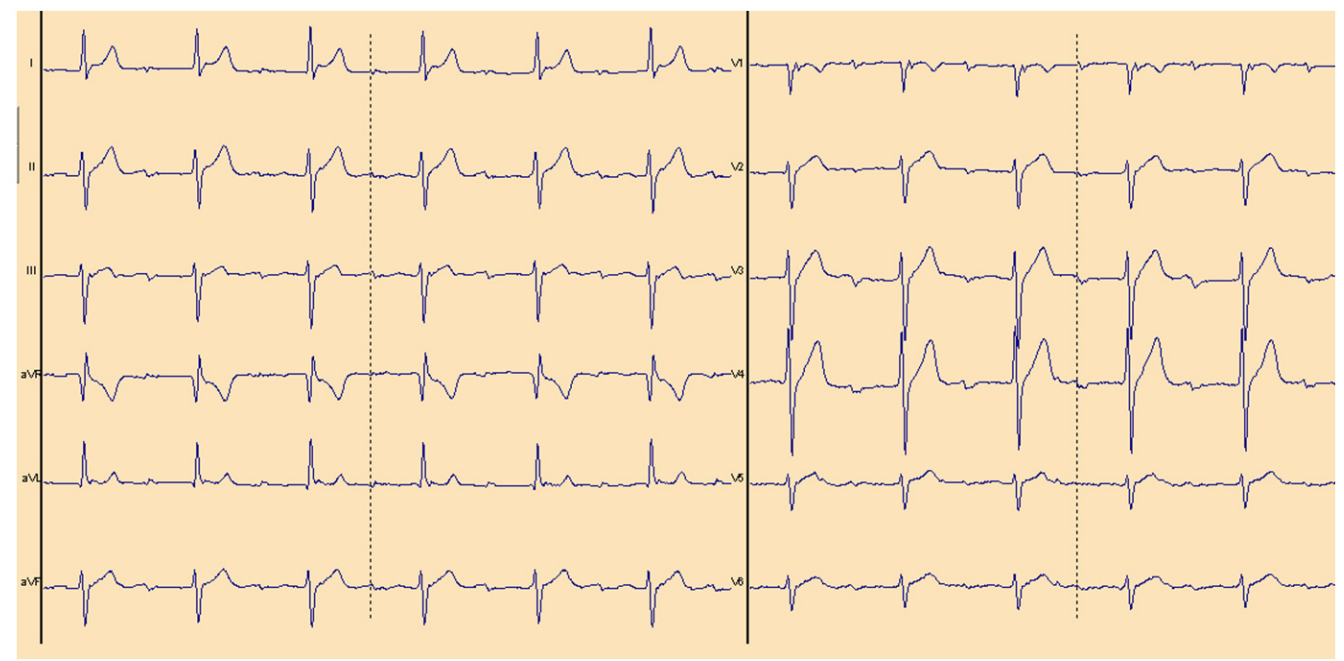

Figure 4. 2:1 AV conduction 12 lead

\section{Discussion}

Focal AT is relatively rare, accounting for between 3 and 17 percent of arrhythmias in adults, which participate to studies for paroxysmal supraventricular tachycardia. ${ }^{[1]}$ It is defined as a regular atrial rhythm that arise outside of the sinus node, from a single site within the left or right atrium, which may be due either by a micro reentry or by the exaltation of the automatism of an ectopic focus and may also result from triggered activity. ${ }^{[2]}$ Although AV conduction is usually 1:1 and the PR interval is often in the normal range producing a long R-P interval, occasionally, especially when the heart rate is particularly high or the AV conduction is markedly increased, ${ }^{[3]}$ a focal AT with a 1:1 AV conduction may have a short RP interval $(<90 \mathrm{~ms})$. In these cases it is necessary to differentiate AT from other SVTs and in particular from the typical AVNRT, that is one of the most common paroxys- mal SVTs ${ }^{[1]}$ and that is a regular SVT. Normally it initiate with a premature atrial beat or more rarely with a ventricular premature beat, and it has a "short RP" interval because the atrial activation coincide with ventricular activation or follow it with a short time and thus, on electrocardiographic trace, $\mathrm{P}$ wave is placed nearer the previous QRS complex than the following one. In these cases, it is easily understandable that the differential diagnosis between the two forms of tachycardia can be particularly difficult. Nevertheless, differential diagnosis is important because the ongoing management of SVTs is different depending on the type of arrhythmia, for example the catheter ablation is considered a reasonable alternative to oral drug therapy with beta-blockers and calcium antagonists in patients with focal AT symptomatology, while in subjects with symptomatic AVNRT represents the first-line procedure ${ }^{[1]}$ Differential diagnosis can be obtained by elec- 
trocardiographic analysis, medical therapy including vagal maneuvers and adenosine infusion and electrophysiologic (EP) study . The EP study allows to achieve the correct diagnosis of SVT, identifying the source site and the mechanism behind the tachyarrhythmia through the cardiac mapping performed during the procedure. Furthermore, it permits a definitive therapy if accompanied by catheter ablation. ${ }^{[1,2]}$ However, it is an invasive test and it was not performed in our patient considering her age and comorbidity. Therefore, we preferred to achieve further additional details to distinguish AT from other SVTs through the non-invasive Holter ECG analysis. Indeed, the monitoring of the electrocardiographic trace within 24 hours, may highlight suggestive clues of AT such as a Wenckebach conduction during a SVT ${ }^{[4-6]}$ and the slight variation of PP and RR intervals. ${ }^{[2]}$

\section{CONFLiCTS OF InTEREST Disclosure}

The authors have declared no conflicts of interest.

\section{REFERENCES}

[1] Page RL, Joglar JA, Caldwell MA, et al. Evidence Review Committee Chair. 2015 ACC/AHA/HRS Guideline for the Management of Adult Patients With Supraventricular Tachycardia: A Report of the American College of Cardiology/American Heart Association Task Force on Clinical Practice Guidelines and the Heart Rhythm Society. Circulation. 2016 Apr 5; 133(14): e506-74. Epub 2015 Sep 23. PMid:26399663 https://doi.org/10.1161/CIR. 0000000000 000311

[2] Chen SA, Chiang CE, Yang CJ, et al. Sustained atrial tachycardia in adult patients. Electrophysiological characteristics, pharmacological response, possible mechanisms, and effects of radiofrequency ablation. Circulation. 1994; 90: 1262. PMid:8087935 https : //doi.org/10.1161/01.CIR.90.3.1262

[3] Rosso R, Kistler PM. Focal atrial tachycardia. Heart. 2010; 96: 181-5.
PMid:19443472 https://doi.org/10.1136/hrt.2008.14355 2

[4] Oreto G. I disordini del ritmo cardiaco. Torino, Centro Scientifico Editore; 1997.

[5] Carbone V, Pia Calabrò M, Marafioti V, et al. Atrial tachycardia with Wenckebach atrioventricular conduction mechanism: What is the origin of the beat following the pause? Heart Rhythm. 2016 Jan; (1): 321-3. PMid:26723506 https://doi .org/10.1016/j.hrth m.2015.10.009

[6] Buttà C, Tuttolomondo A, Giarrusso L, et al. Electrocardiographic Diagnosis of Atrial Tachycardia: Classification, P-Wave Morphology, and Differential Diagnosis with Other Supraventricular Tachycardias. Ann Noninvasive Electrocardiol. 2015 Jul; 20(4): 314-27. Epub 2014 Dec 22. PMid:25530184 https://doi.org/10.1111/anec. 122 46 